\title{
Dynamically Enlarge and Shrink Power Coverage to Speed Up Tag Identification in an RFID System
}

\author{
Tsang-Ling Sheu, Jian-Xing Zhu \\ Department of Electrical Engineering, National Sun Yat-sen University, Taiwan \\ Email: sheu@ee.nsysu.edu.tw
}

How to cite this paper: Sheu, T.-L. and Zhu, J.-X. (2018) Dynamically Enlarge and Shrink Power Coverage to Speed Up Tag Identification in an RFID System. Journal of Computer and Communications, 6 , 247-263.

https://doi.org/10.4236/jcc.2018.611023

Received: October 25, 2018

Accepted: November 25, 2018

Published: November 28, 2018

Copyright (c) 2018 by authors and Scientific Research Publishing Inc. This work is licensed under the Creative Commons Attribution International License (CC BY 4.0).

http://creativecommons.org/licenses/by/4.0/

\begin{abstract}
In this paper, we present a power adjustment scheme to dynamically enlarge and shrink power coverage to speed up tag identification in an RFID system. By dividing a TDMA frame into time slots, the proposed power adjustment scheme can adaptively increase or decrease the transmission power of a reader. Specifically, due to the contention for a TDMA slot from numerous tags, three states of a slot could exist; they are respectively referred to as successful, collided, and idle states. An adjustment factor based on the three states is designed to dynamically adjust the transmission power of a reader. The design of the adjustment factor considers two different aspects. When the number of idle state far exceeds the number of collided state, the first aspect will enlarge the power such that more tags within the coverage can be concurrently identified. On the other hand, when the number of idle state is much smaller than the number of collided state, the second aspect will shrink the power such that the number of tags within the coverage is significantly reduced. The proposed power adjustment scheme is simulated using NS-3. In the simulation, we design three different topologies which place tags in three distributions, uniform, random, and hotspot. From the simulation results, we demonstrate that the proposed power adjustment scheme can speed up the tag identification and save energy consumption, particularly when a large number of tags are placed in hotspot distribution.
\end{abstract}

\section{Keywords}

RFID, TDMA, Tag Identification, Dynamic Power Adjustment, NS-3

\section{Introduction}

Along with the increasing requirements for facilitating the convenience in our 
living environments, the technology of RFID (Radio Frequency Identification) has been widely deployed. An RFID system consists of a reader and a large number of tags. There are many existing applications using RFID today, for examples, tagging a vehicle for electronic toll collections (ETC), tagging a passing card for subways, tagging merchandise in supermarkets, etc. In these applications, a reader usually needs to read hundreds or thousands of tags' ID in a short time. Yet, simultaneously reading more than one tags' ID may create collisions in a reader system. Thus, in recent years, how to effectively and speedily read tags' ID form a reader has become an important research subject.

Differentiated by the media access control, previous work on resolving the collisions in an RFID system can be divided into four categories, FDMA (Frequency Division Multiple Access), CSMA (Carrier Sense Multiple Access), SDMA (Space Division Multiple Access), and TDMA (Time Division Multiple Access). In FDMA, multiple channels in terms of different frequencies are used among different readers to avoid collisions. For example, Yu, et al. [1] assumed that a reader before it begins to read tags' ID would issue a beacon message to alarm the neighbor readers. In CSMA, a tag will detect the busy or idle state of a carrier before it can transmit back its ID to the reader. For example, Leonardo et al. [2] proposed a $P$-persistent scheme to resolve the collisions among tags, where $P$ is the probability for a tag to begin to transmit or continue to listen when an idle state of a carrier is detected. In SDMA, directional antenna was used to divide the space into different sectors such that the collisions of the tags in different sectors can be avoided. For example, Kim et al. [3] proposed a method for a multi-antenna reader to confirm the successful reading when it reads tags' ID in different sectors.

Besides the above mentioned three categories, TDMA noticeably attracts the most attentions to researchers. A TDMA frame consists of multiple slots where a tag uses a slot (considered as a channel) to transfer back its ID to a demanding reader. Yet, collisions may occur when more than one tag pick up the same slot. Most of the researchers in TDMA therefore work on a model to estimate the appropriate number of slots in a frame based on different levels of collisions. For examples, Onat, et al. [4] proposed a polynomial theorem which can estimate the number of slots in a frame suitable for reading a large number of tags. Luo, et al. [5] proposed a method which can estimate the number of unread tags based on the ratio of collision slots. Similarly, Zhu, et al. [6] built a Markov chain model to estimate the number of unread tags in a frame. Zanella, et al. [7] proposed a method to dynamically adjust the frame size to cope with the number of tags, assuming that the tags would follow Poisson arrivals. Xu, et al. [8] proposed an improved RFID anti-collision algorithm (IAA), which adjusts the frame size based on the number of collided tags in a slot. Wang, et al. [9] proposed a method using the minimized mean square error to estimate the number of tags for determining an appropriate frame size. Chen, et al. [10] proposed a lottery frame (LoF), which can estimate the number of tags to effectively reduce the tags' identification time. Other researchers focused on a tree-splitting algo- 
rithm, which estimates the number of tags to dynamically adjust the frame size. For examples, Porta, et al. [11] [12] proposed a binary-tree splitting to estimate the number of tags. Wu, et al. [13] proposed a $Q$-ary tree splitting algorithm, which can compute the value of $Q$ based on the ratio between the idle and the collision slots. To extend the $Q$-ary tree splitting algorithm, Chen, et al. [14] [15] [16] and Farooq, et al. [17] derived the optimal value of $Q$ through simulation. On the other hand, some researchers adjusted the frame size by dividing the tags into a number of groups. For examples, Qiao, et al. [18] and Wang, et al. [19] proposed a matching and grouping algorithm, which used the average estimate method to divide tags into $n$ groups; each group was adjusted to fit a different frame size. Su, et al. [20] proposed a method using Chinese remainder theory to divide the tags into several overlapping groups. Finally, Tseng, et al. [21] proposed the spread partial-Q slot count (SPSC) algorithm, which divided tags into different sectors based on the receiving power of tags. The authors demonstrated that a reader using SPSC can significantly reduce the collisions when a large number of tags are to be identified.

The previous work resolved the collisions in an RFID system using TDMA either by adjusting the size of a frame or by dividing the tags into different groups. In this paper, without paying the cost in frequently varying the frame sizes or aforetime dividing the tags into several groups, we propose a dynamic power adjustment (DPA) scheme to speed up the tag identification time. The proposed DPA increases or decreases the transmission power of a reader based on three states (successful, collided, and idle) of a slot in a TDMA frame. Two aspects are considered in adjusting the transmission power. The first aspect increases the power to read more tags, if the number of idle state far exceeds the number of collided state. The second aspect cut power to reduce the number of readings, if the number of idle state is much smaller than the number of collided state.

The remainder of this paper is organized as follows. In Section 2, an RFID system and the format of a tag's ID are introduced. In Section 3, we propose a dynamic power adjustment scheme for a reader to speed up the tag identification time. In Section 4, simulation on NS-3 is performed and the results are discussed. Finally, conclusion remarks are drawn in Section 5.

\section{An RFID System}

Figure 1 shows an application of an RFID system. The system consists of a reader, numerous tags, and an Internet server associated with a database. The reader periodically issues a broadcasting request message using radio frequency (RF) to all the passive tags. Once receiving the request, a tag should respond with its unique ID by picking up a slot in a TDMA frame. After successfully reading a tag's ID, the reader will send it, over wired or wireless media, to an Internet server, which stores the tag's ID in a database for the future use.

Figure 2 shows the format of a tag ID, defined in EM 4100 protocol [22]. The ID consists of 64 bits using Manchester codes. The first 9 bits are all 1's, 
representing the start communication bits. D00 to D07 are the version number or customer ID. D08 to D39 are the 32-bit unique ID for a tag. Using even parity, P0 to P9 are the row parity bits, while PC0 to PC3 are the column parity bits. At last, S0 (set to 0 ) is the stop bit of the ID.

\section{Dynamic Power Adjustment}

\section{1) Three States of a Slot}

As shown in Figure 3, in the proposed DPA, we design TDMA frames, appearing from Frame 0 to Frame $n$ for a reader to quickly identify tags. A TDMA frame consists of $m$ slots, numbered from 0 to $m$-1, and followed by a bit map with $m$ bits. The size of each slot is large enough to accommodate the $k$ bits of a Tag ID; for example, $k=64$ bits in EM 4100. A slot has three states, successful (in blue color), collided (in red color), and idle (in white color). A successful state denotes that a slot is picked by exactly one tag. Thus, a tag ID can be successfully read because no collision occurs. On the contrary, a collided state cannot return any tag ID to the reader since more than one tag pick the same slot. An idle state simply denotes a state without tag selection. To inform a tag whether the selection of a slot is successful or collided, we design the $m$-bit map, where 0 denotes the selection of a slot is successful and 1 denotes the selection is unsuccessful (or collided). Notice that initially Frame 0 carries only the $m$-bit map with all bits being set to 1 . Let's use Frame 1 as an example. Slot 0 is in the successful state (in blue color), since it is picked by exactly one tag. Hence, the first bit of the bit map is 0 . However, slot 1 is in the collided state (in red color), since it is picked by more than one tag. Thus, the second bit of the bit map is 1 .

2) Factor to Adjust the Power

To reduce the collisions, the proposed DPA increases or decreases the transmission power of a reader based on the three states of a slot. In other words, reducing transmission power of a reader will reduce the coverage. As a result, the number of tags to be identified is reduced. On the other hand, increasing transmission power of a reader will increase the coverage, which in turn increases the probability of collisions. Figure 4 illustrates the transmission power of a reader versus the coverage of tags. Table 1 shows the parameters and their definitions used in the DPA.

Based on the three states of a slot, a factor $(F)$ to adjust the transmission power is computed as shown in Equation (1), where $I$ denotes the number of idle slot, $C$ denotes the number of collided slot, and $S$ denotes the number of successful slot. Specifically, if $F$ is positive and greater than one, then the transmission power of a reader is enlarged by multiplying $F$. Notice that the second term in Equation (1) is always greater than one. Thus, if $I$ is greater than $C$, then $F$ should be greater than one.

$$
F=\left(\frac{I}{C}\right) \times\left(\frac{S}{S+C}\right)
$$

Equation (2) is used to compute the transmission power $\left(P_{n+1}\right)$ of a reader at 
Frame $n+1$ from the transmission power $\left(P_{n}\right)$ of a reader at Frame $n$. Since any one of the three parameters, $S, C$, and $I$, could be zero, we have to design another four parameters, $\Delta_{1}, \Delta_{2}, \Delta_{4}$, and $\Delta_{4}$, as shown in Equation (3), for power adjustment. In Equation (3), $m$ is the total number of slot in a frame and $P_{0}$ is the minimum transmission power of a reader. $\Delta_{1}$ is positive or negative depending on whether $F$ is greater than or smaller than one. $\Delta_{2}$ is always positive and it is designed when $C=0$ and $I \neq 0$. To the contrast, $\Delta_{3}$ is always negative and it is designed when $C \neq 0$ and $I \neq 0$. Finally, $\Delta_{4}$ is positive or negative depending on whether $I$ is greater than or smaller than $C$. It is noticed that $P_{n+1}$ is no change from $P_{n}$ as long as $S \neq 0, C=0$, and $I=0$.

$$
\begin{aligned}
& P_{n+1}=\left\{\begin{array}{l}
P_{n}+\Delta_{1}, \text { if } S \neq 0 \text { and } C \neq 0 \text { and } I \neq 0 \\
P_{n}+\Delta_{2}, \text { if } C \neq 0 \text { and } I \neq 0 \\
P_{n}+\Delta_{3}, \text { if } C \neq 0 \text { and } I=0 \\
P_{n}+\Delta_{4}, \text { if } S=0 \text { and } C \neq 0 \text { and } I \neq 0 \\
P_{n}, \text { if } S \neq 0 \text { and } C=0 \text { and } I=0
\end{array}\right\} \\
& \left\{\begin{array}{l}
\Delta_{1}=(F-1) \times P_{0} \\
\Delta_{2}=\frac{I}{m} \times \frac{I}{S+I} \times P_{0} \\
\Delta_{3}=-\left(\frac{C}{m} \times \frac{C}{S+C} \times P_{0}\right) \\
\Delta_{4}=\frac{I-C}{m} \times P_{0}
\end{array}\right\}
\end{aligned}
$$

We illustrate the proposed DPA with two scenarios, as shown in Figure 4(a) and Figure 4(b), respectively for the decrease and increase of the transmission power of a reader. In Figure 4(a), since the number of collided slot far exceeds the number of idle slot, from Equation (1) we know $F$ should be smaller than one. From Equation (3), we know $\Delta_{1}$ becomes negative. Thus, from Equation (2), $P_{n+1}$ is decreased from $P_{n}$ by deducting $\Delta_{1}$. On the other hand, in Figure 4 (b), since the number of idle slot far exceeds the number of collided slot, from Equation (1) we know $F$ is greater than one. From Equation (3), we know $\Delta_{1}$ becomes positive. Thus, from Equation (2), $P_{n+1}$ is increased from $P_{n}$ by adding $\Delta_{1}$.

Figure 5 shows the operation flow of a tag. When a frame arrives, a tag examines whether the bit (representing the chosen slot in the bit map) is one; if it is not one, the tag's ID is successfully transmitted to the reader; otherwise, the tag will have to choose a slot again in Frame $n+1$.

Figure 6 shows the operation flow of a reader. When a frame (Frame $n$ ) begins, the reader examines the status of every slot in the frame. Depending on the slot status, the reader marks $m$ bits one by one in the bit map; it marks a bit as one if a collided slot, and zero if a successful slot. The reading of tag's ID is completed, if all slots are idle; otherwise, the reader will examine the three parameters, $S, C$, and $I$, for the corresponding power adjustments, as described in Equation (2) and Equation (3). 


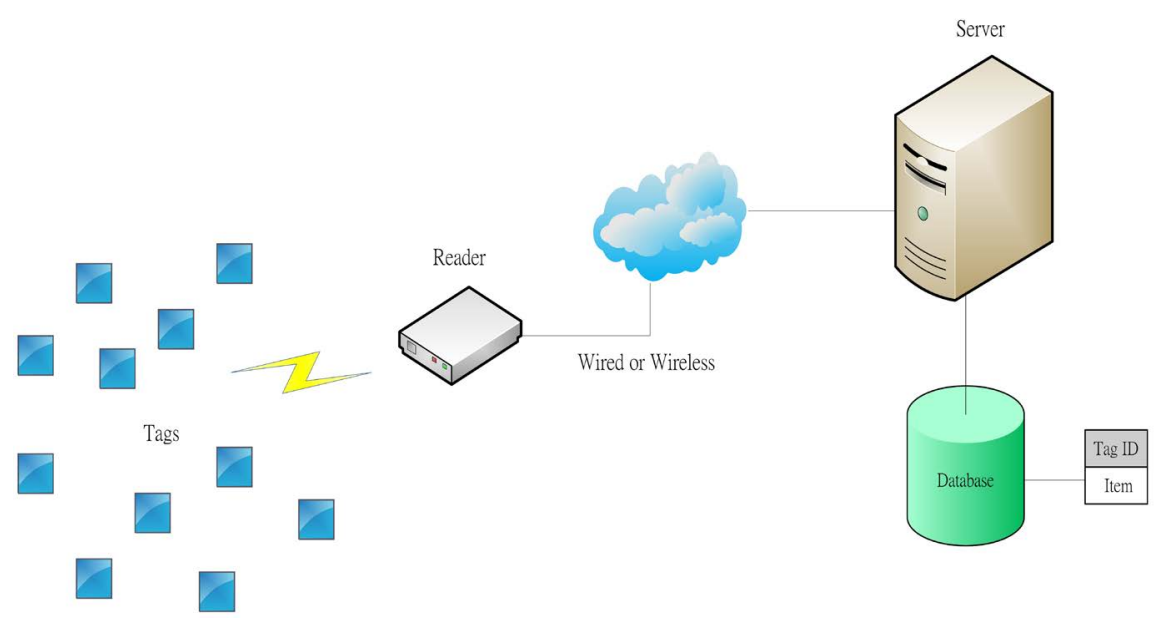

Figure 1. A reader and numerous tags in an RFID system.

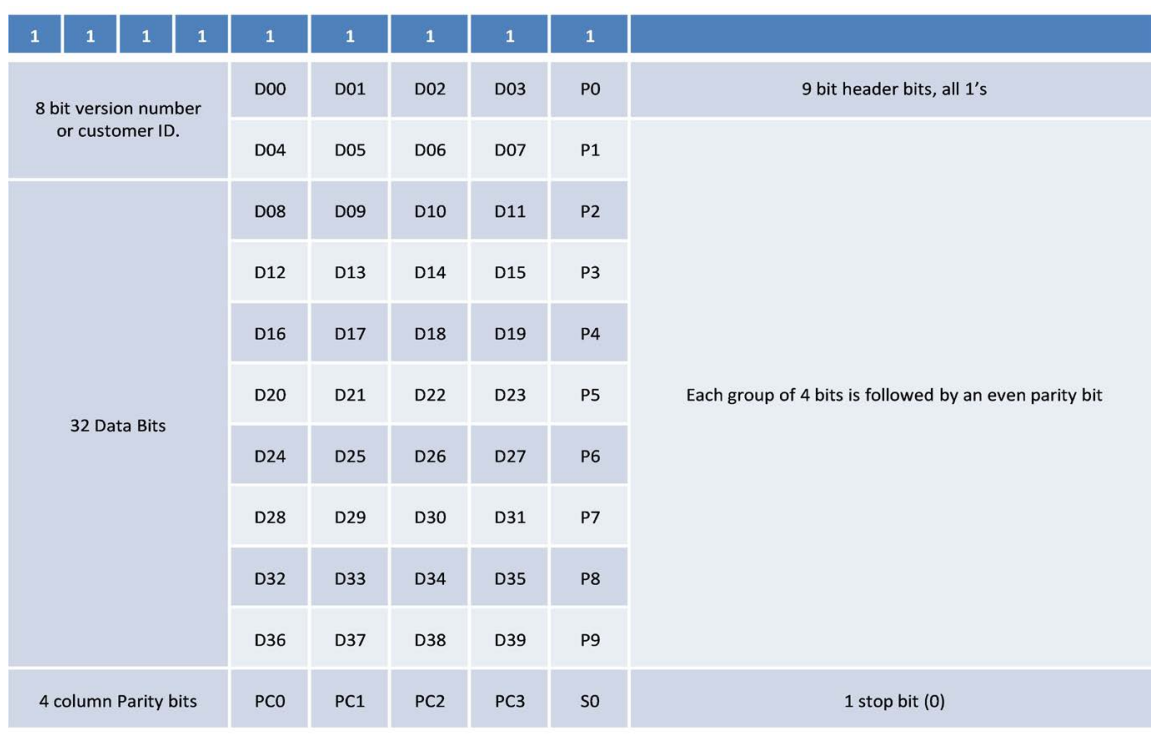

Figure 2. Format of a Tag ID.

Frame 0

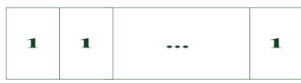

Frame 1

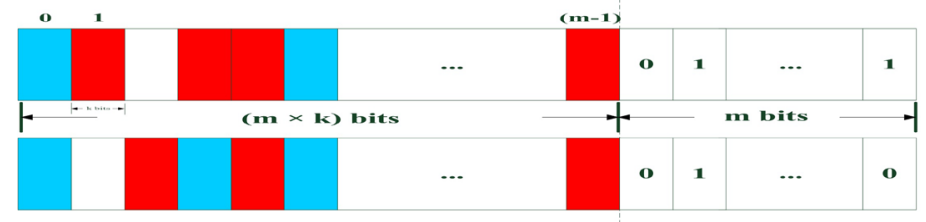

Framen

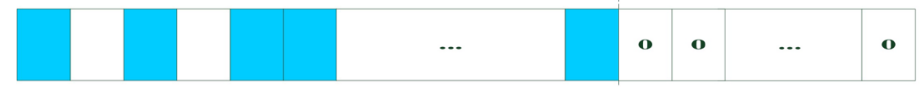

Figure 3. Three states of a slot in a TDMA frame. 


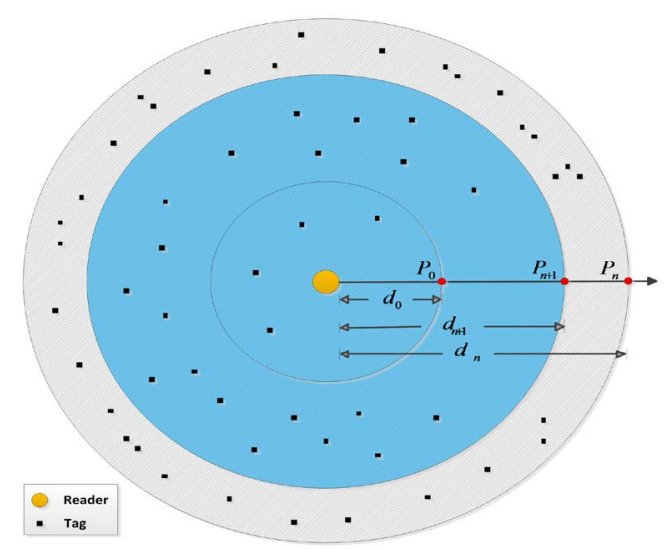

(a)

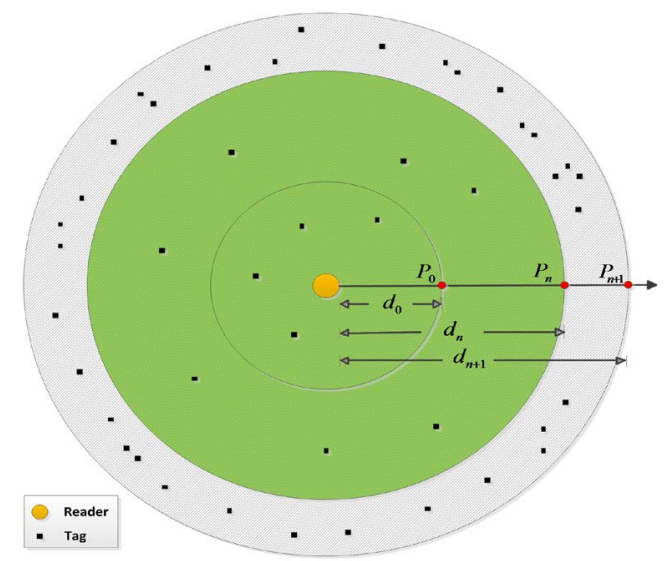

(b)

Figure 4. Two different scenarios of the DPA. (a) Decrease of the transmission power; (b) Increase of the transmission power.

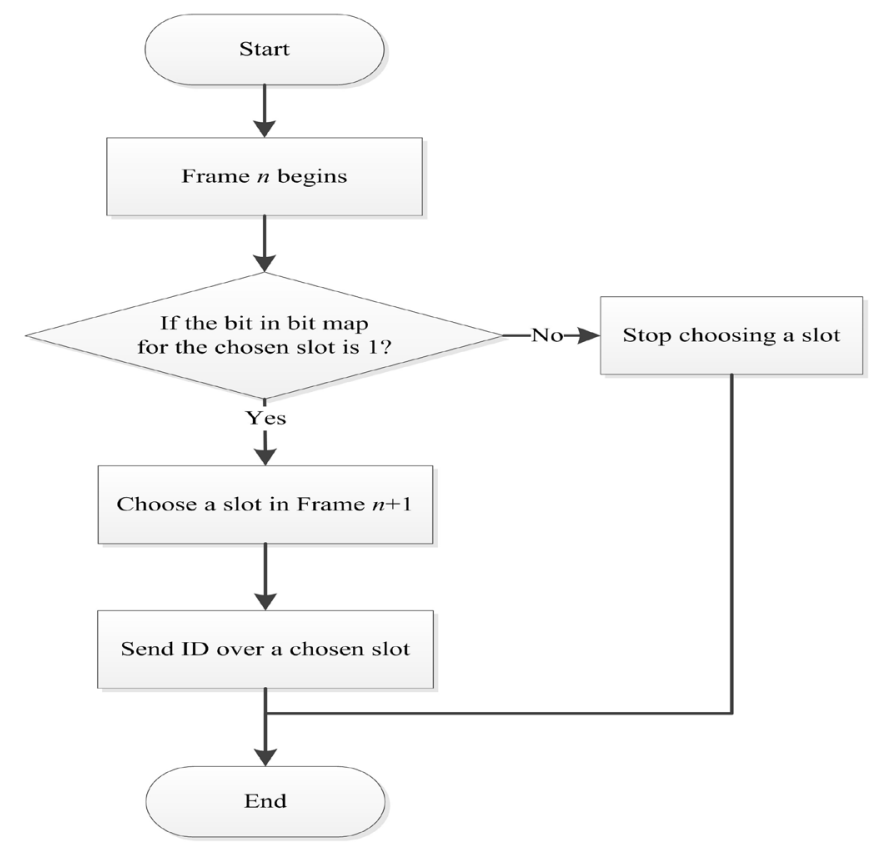

Figure 5. The operation flow of a tag. 


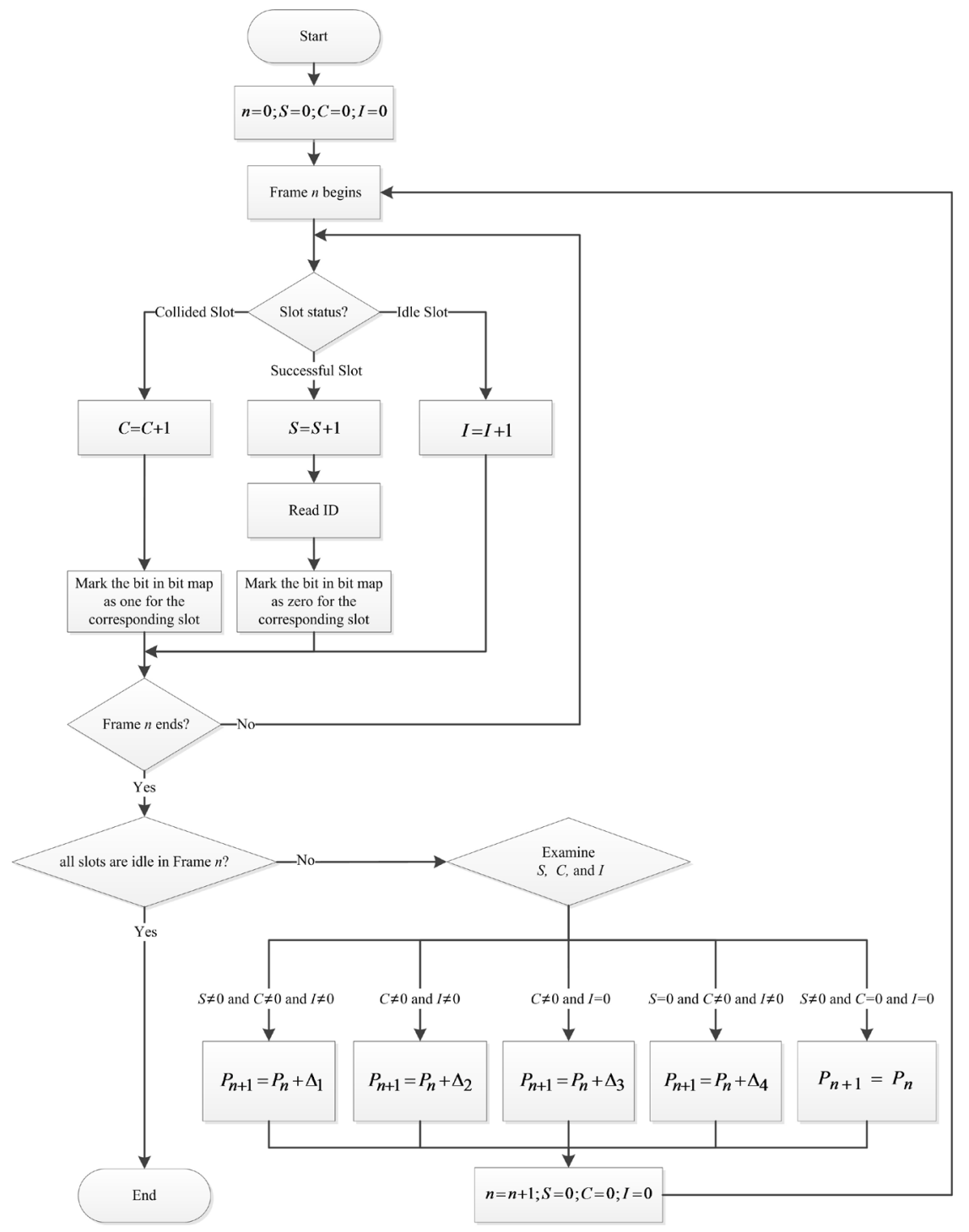

Figure 6. The operation flow of a reader.

Table 1. Parameters and definitions.

\begin{tabular}{cc}
\hline Parameter & Definition \\
\hline$S$ & Successful slots in a TDMA frame \\
$I$ & Collided slots in a TDMA frame \\
$F$ & Idle slots in a TDMA frame \\
$P_{0}$ & Power adjustment factor \\
$P_{n}$ & Minimum transmission power of a reader \\
$P_{n+1}$ & Transmission power in the $n$-th frame \\
$d_{0}$ & Transmission power in the $(n+1)$-th frame \\
$d_{n}$ & Radius of a reader's minimum coverage \\
$d_{n+1}$ & Radius of a reader's coverage in the $n$-th frame \\
\hline
\end{tabular}




\section{Simulation and Discussions}

To analyze the proposed DPA in terms of tag identification time, the number of undetected tags, and total energy consumption, we perform simulation using NS-3 with an embedded RFID module. As illustrated in Figure 7, a tag consists of transmit/receive module, slot-choosing module, and a reader consists of receiving module, DPA module, and power control module.

To analyze different placements of tags, three topologies of tag distribution, uniform, random, and hotspot, are designed in the NS-3 simulation, as shown in Figure 8.

We define the transmission power $\left(P_{t}\right)$ of a reader in Equation (4) and the energy consumption $(E C)$ of a reader in Equation (5). In Equation (4), $P_{r}$ is the receiving power of a tag, $d$ is the distance between a tag and its reader, and $\lambda$ is the wavelength for radio. In Equation (5), $T$ is the total operation time of a reader. Table 2 shows the parameters and their settings used in the simulation.

Table 2. Parameters and settings in the simulation.

\begin{tabular}{cc}
\hline Parameters & Settings \\
\hline$P_{0}$ (Minimum transmission power) & $0.05 \mathrm{~mW}$ \\
$P_{r}$ (Receiving power) & $3.65 \times 10^{-7} \mathrm{~mW}$ \\
$P_{\max }$ (Maximum transmission power) & $0.53 \mathrm{~mW}$ \\
$\Lambda$ (Wavelength) & 0.33 meters \\
$m$ (Slots in a frame) & 16 or 64 slots \\
\hline
\end{tabular}

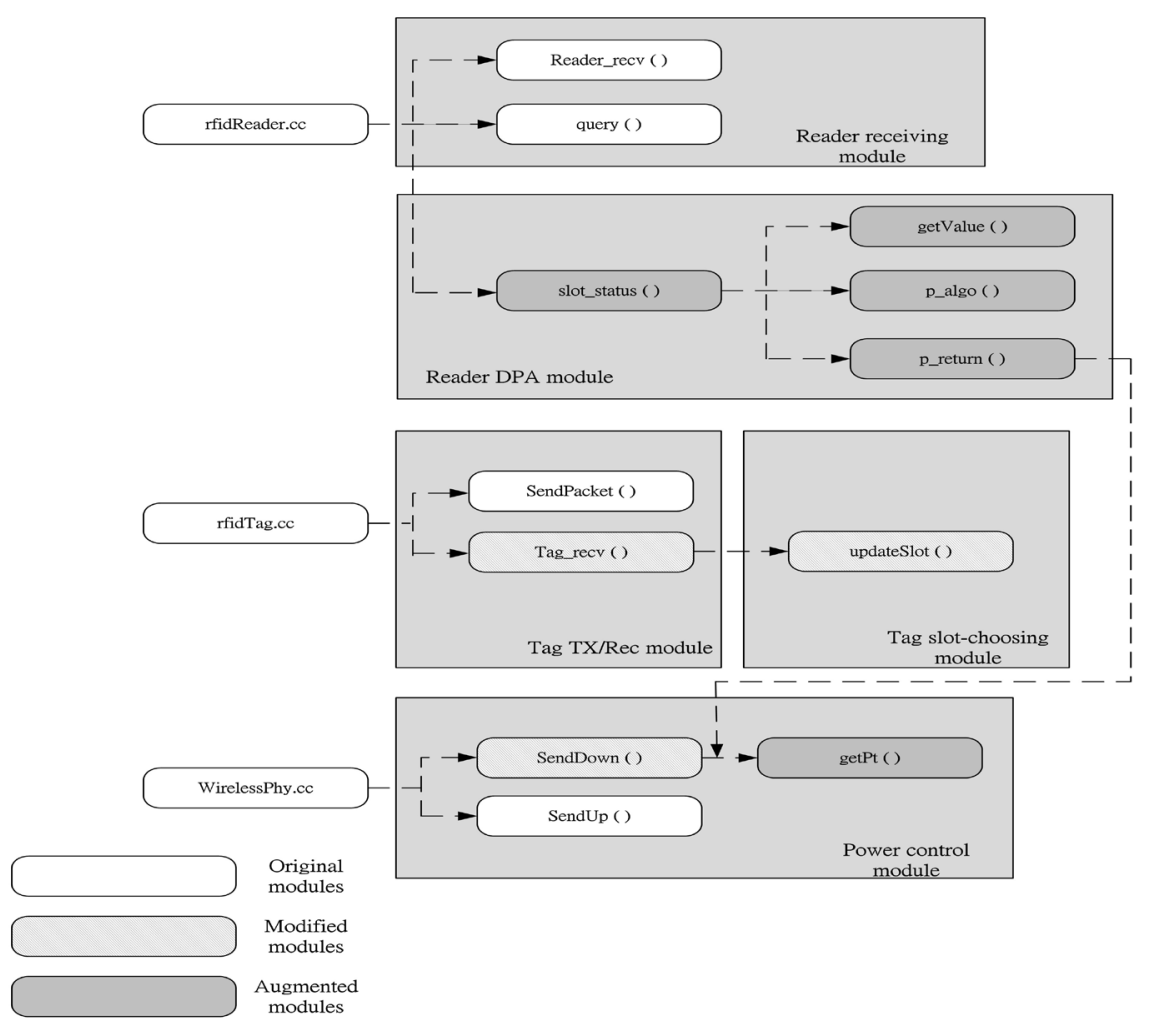

Figure 7. The proposed DPA with RFID modules in NS-3. 


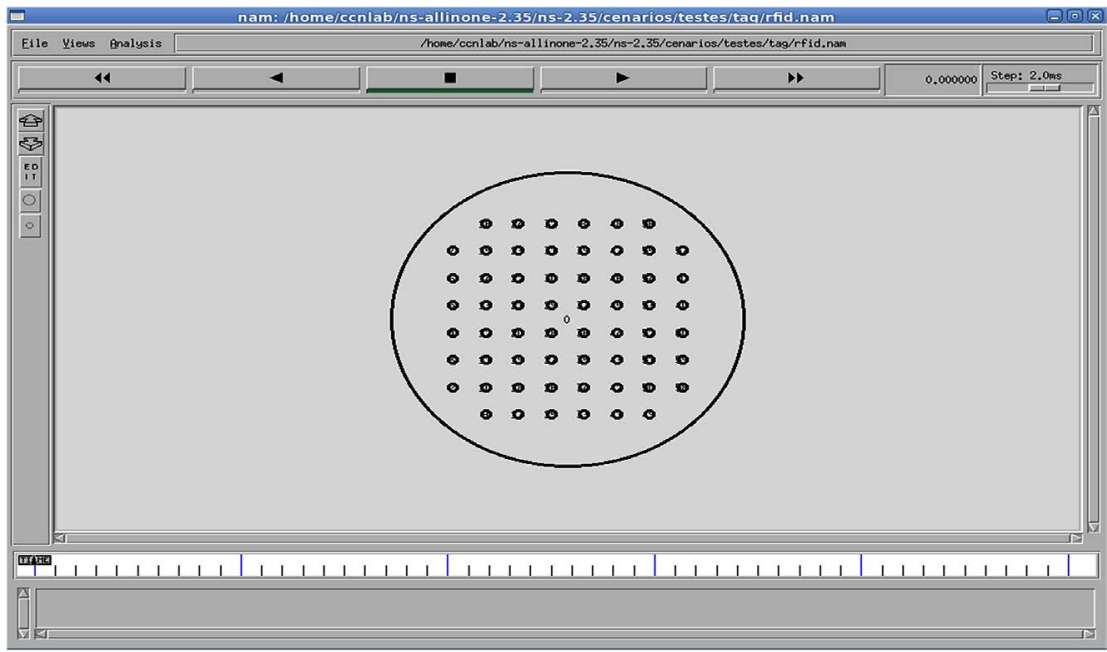

(a)

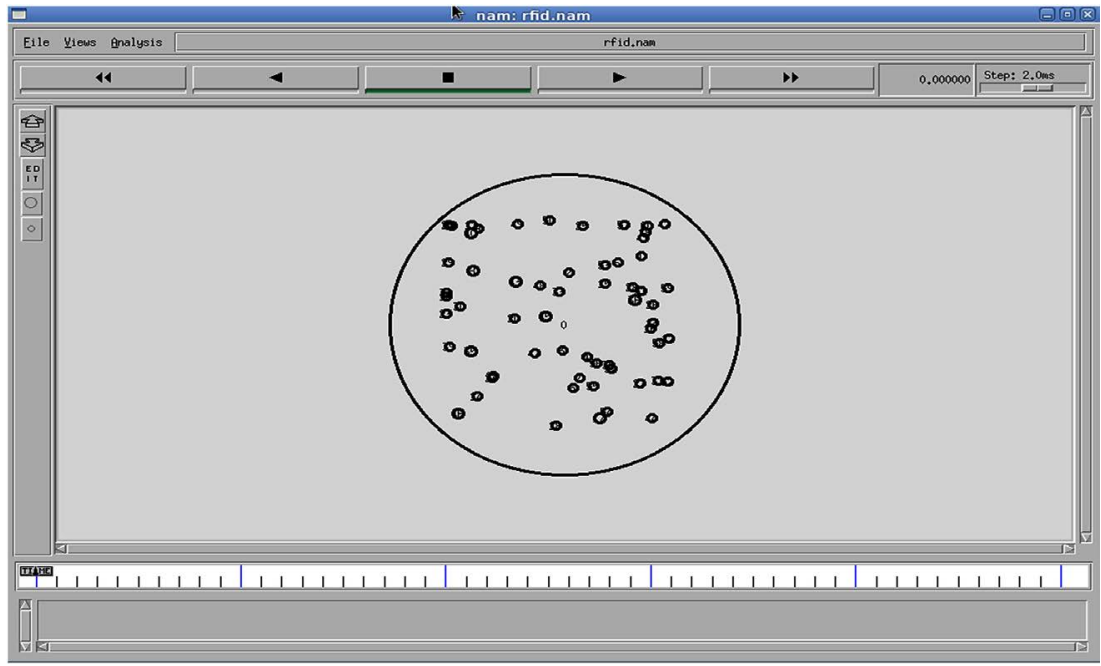

(b)

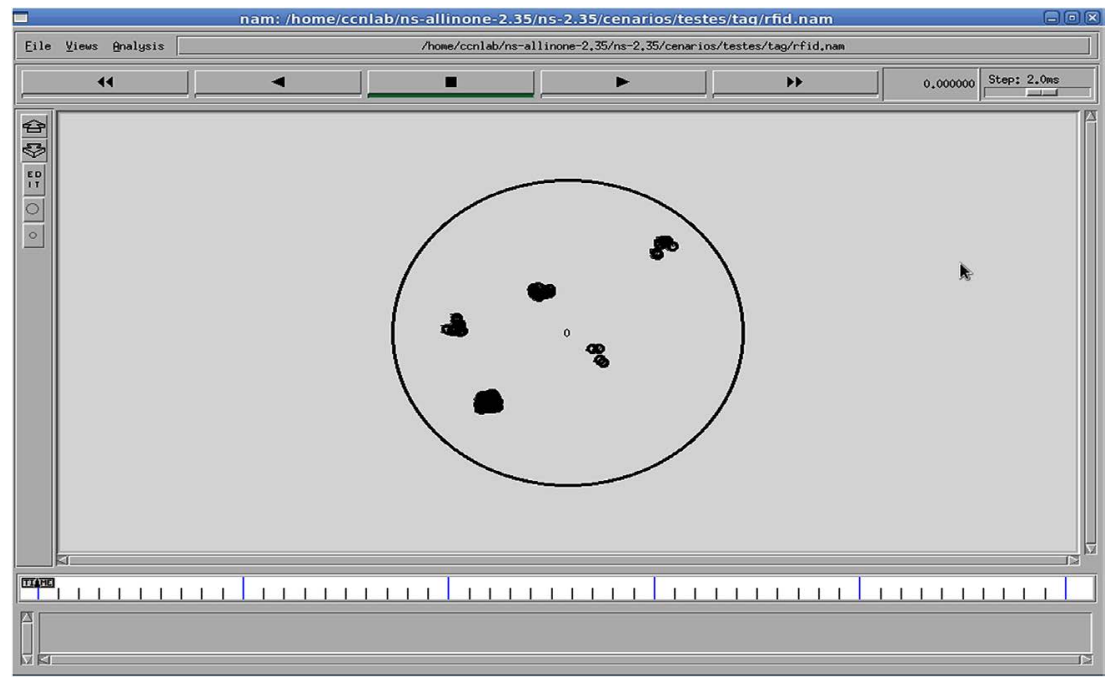

(c)

Figure 8. Three different topologies of tag distribution. (a) Uniform distribution; (b) Random distribution; (c) Hotspot distribution. 


$$
\begin{gathered}
P_{t}=\frac{P_{r} \times(4 \pi d)^{2}}{\lambda^{2}} \\
E C=P_{t} \times T
\end{gathered}
$$

From Equation (4) and by substituting $P_{t}=0.53 \mathrm{~mW}, P_{r}=3.65 \times 10^{-7}$ $\mathrm{mW}, \lambda=0.33$ meters, we can compute the maximum distance $\left(d_{\max }\right)$ between a reader and a tag, which is about 10 meters. In other words, we assume the maximum coverage of a reader is 10 meters in the simulation.

By considering the three different topologies of tag distribution, Figure 9(a), Figure 9(b), and Figure 9(c) compares the number of frames used in a reader between the proposed DPA and a previous work SPSC [21], which divides the tags into 4 (SPSC-4) and 10 (SPSC-10) groups, respectively. As it can be observed, although SPSC-4 spends only 5 frames to read 10 tags, the proposed DPA spends the fewest frames to read tags increasing from 20 to 60, no matter which tag distribution is applied. In particular, when the number of tag is increased to 60 , the proposed DPA significantly outer-performs both SPSC-4 and SPSC-10 in reducing the tag identification time. It is also noticed that, unlike SPSC- 4 or SPSC-10, the number of frames spent in reading all tags by using the proposed DPA does not increase largely along with the increase of the number of tags. It is also interesting to observe that the curve of the number of frames spent versus the number of tags (increasing from 10 to 60) is even more flat when the tags are placed in hotspot distribution.

Since a reader in SPSC consistently maintains its transmission power in the maximum level, it will consume too much power. To the contrast of SPSC, we introduce two variation versions of the proposed DPA to significantly save the transmission power of a reader. The first variation is referred to as DPA with proportionally increasing power from the minimum to the maximum (PIP-MIM). The second variation is referred to as DPA with proportionally decreasing power from the maximum to the minimum (PDP-MAX).

Although both PIP-MIM and PDP-MAX can greatly reduce the power consumed in a reader, the later version (PDP-MAX) may not $100 \%$ read all the tags, since some tags may remain unidentified between the outer coverage when a larger transmission power is used and the inner coverage when a smaller transmission power is adjusted.

By considering 100 tags placed in the three distributions, Figure 10(a), Figure 10(b), and Figure 10(c) compare the number of undetected tags between the proposed DPA and its variation, PDP-MAX. In PDP-MAX, the transmission power is decreased in every frame by proportionally multiplying a factor of 0.9 , 0.5 , and 0.1 , respectively. Since the proposed DPA can dynamically increase or decrease the transmission power of a reader based on the three states of a slot, it is observed that all the tags can be read without any missing. However, PDP-MAX has left a lot of tags unread. It is interesting to notice that decreasing the power proportionally by a factor from 0.1 to 0.9 does not make big difference in the number of unread tags when the tags are placed in uniform distribution. Yet, it does improve to reduce the number of unread tags in both random and 
hotspot distributions. Specifically, as the number of tags is increased to 100, in these two distributions, using a larger percentage of power adjustment (i.e., power $\times 0.9$ ) can greatly reduce the percentage of unread tags to $30 \%$ (in random distribution) and $38 \%$ (in hotspot distribution), as it is compared to that by using a smaller percentage of power adjustment (i.e., power $\times 0.1$ ).

From Equation (4) and Equation (5), we can analyze and compare the energy consumption ( $E C$ ) among SPSC, PDP-MAX, PIP-MIM, and the proposed DPA. However, it is very straightforward to announce that the $E C$ in SPSC should be the largest one, since the transmission power of a reader always remains in the maximum. Additionally, we have no doubt that the $E C$ in PDP-MAX should be the smallest one, since it proportionally reduces the power from the maximum to the minimum in every frame. Yet, from Figure 10, the vast drawback in PDP-MAX is that a large percentage of tags may remain unread. Hence, in Figure 11 we would just compare the $E C$ between the proposed DPA and its variation version, the PIP-MIM.

By increasing the number of tag from 100 to 300, Figure 11(a), Figure 11(b), and Figure 11(c) show the comparison of EC between the proposed DPA and its variation, the PIP-MIM, for the three distributions, respectively. In the PIP-MIM, we proportionally increase the transmission power of a reader from the minimum to the maximum in every frame by two different factors, power $\times 1.3$ and power $\times 1.05$. As it can be observed, in general, the proposed DPA has achieved the lowest $E C$ for the three distributions. It is noticed that although the $E C$ in PIP-MIM with power $\times 1.3$ is slightly smaller than that in DPA when the number of tag is 180 in uniform and 150 in random distribution, the $E C$ in PIP-MIM increases drastically from $2 \mathrm{~mJ}$ to $17 \mathrm{~mJ}$ in hot spot distribution when the number of tag is increased from 100 to 300 . On the other hand, the proposed DPA exhibits its superiority in that the $E C$ increases very smoothly, no matter which tag distribution is applied; for instance, the $E C$ just slightly increases from $2 \mathrm{~mJ}$ to no more than $5 \mathrm{~mJ}$ when the number of tag is increased from 100 to 300 and when they are placed in hotspot distribution.

\section{Conclusions}

In this paper, we have presented a dynamic power adjustment (DPA) scheme for a reader to speed up the tag identification time in an RFID system. The proposed DPA can dynamically increase or decrease the transmission power of a reader based on the three states of a TDMA slot, successful, collided, and idle. One of the major contributions of this paper is right in that without paying the cost of frequently varying the frame sizes and aforetime dividing the tags into different groups, the proposed DPA outer-performs a previous work (SPSC) in reducing the tag identification time. Besides, through NS-3 simulation, we have demonstrated that 1) the proposed DPA can read all the tags under the coverage without any missing as it is compared to a proportionally decreasing power scheme (the PDP-MAX); 2) the proposed DPA consumes significantly less energy as it is compared to a proportionally increasing power scheme (the PIP-MIN). 


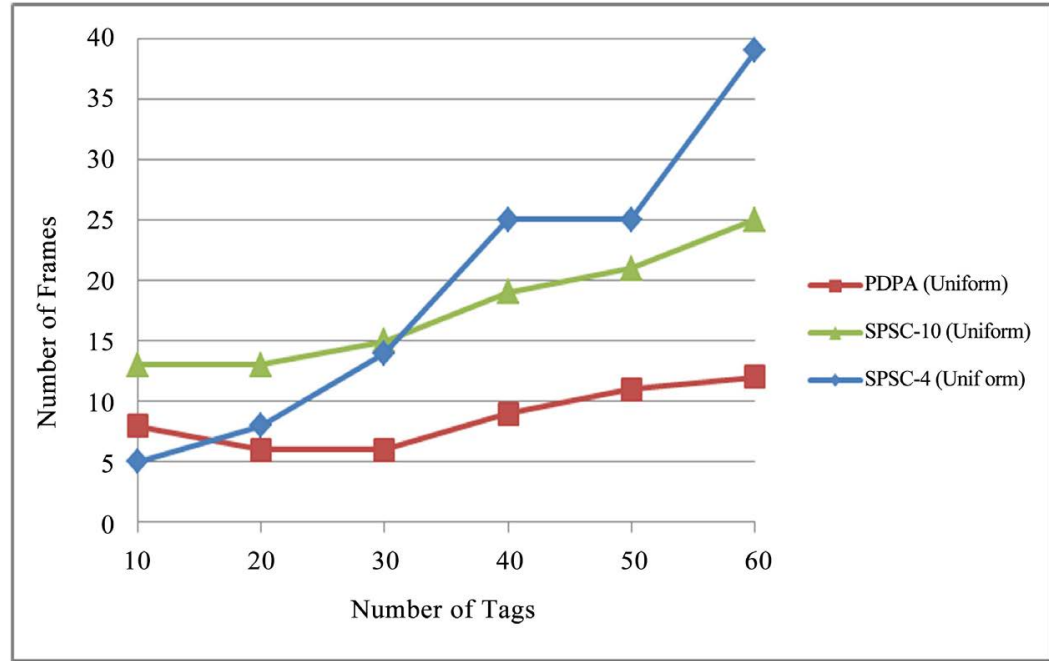

(a)

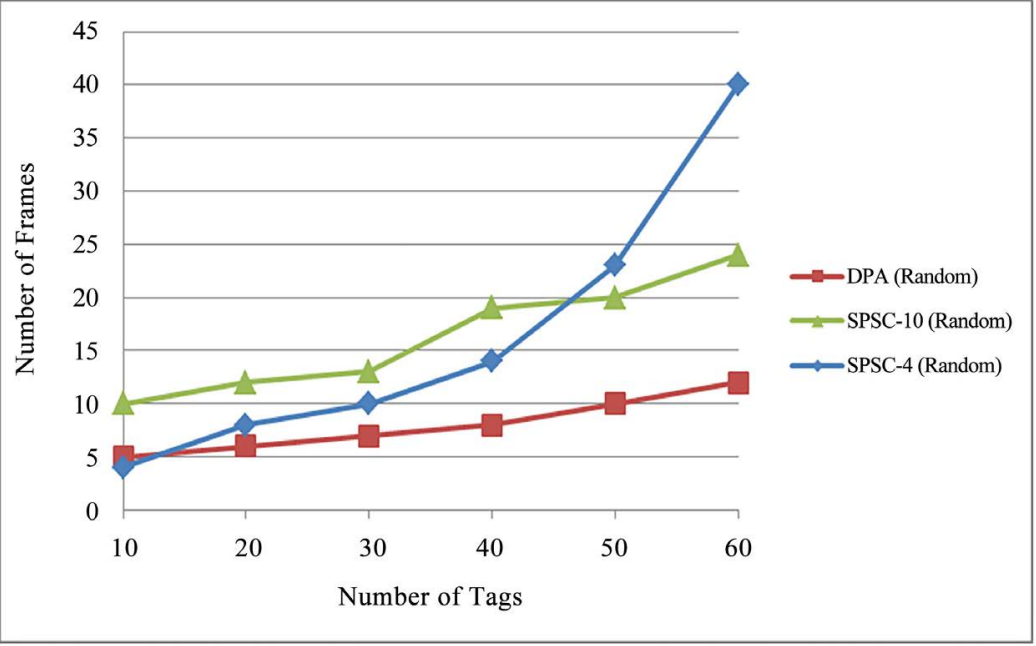

(b)

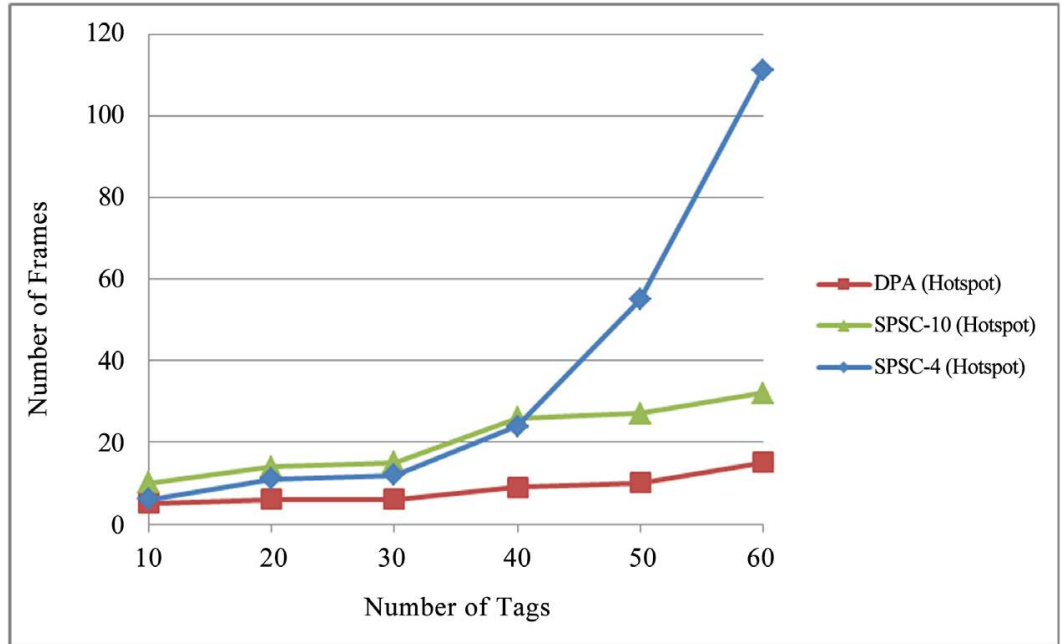

(c)

Figure 9. Number of frames used for three distributions. (a) Uniform distribution; (b) Random distribution; (c) Hotspot distribution. 


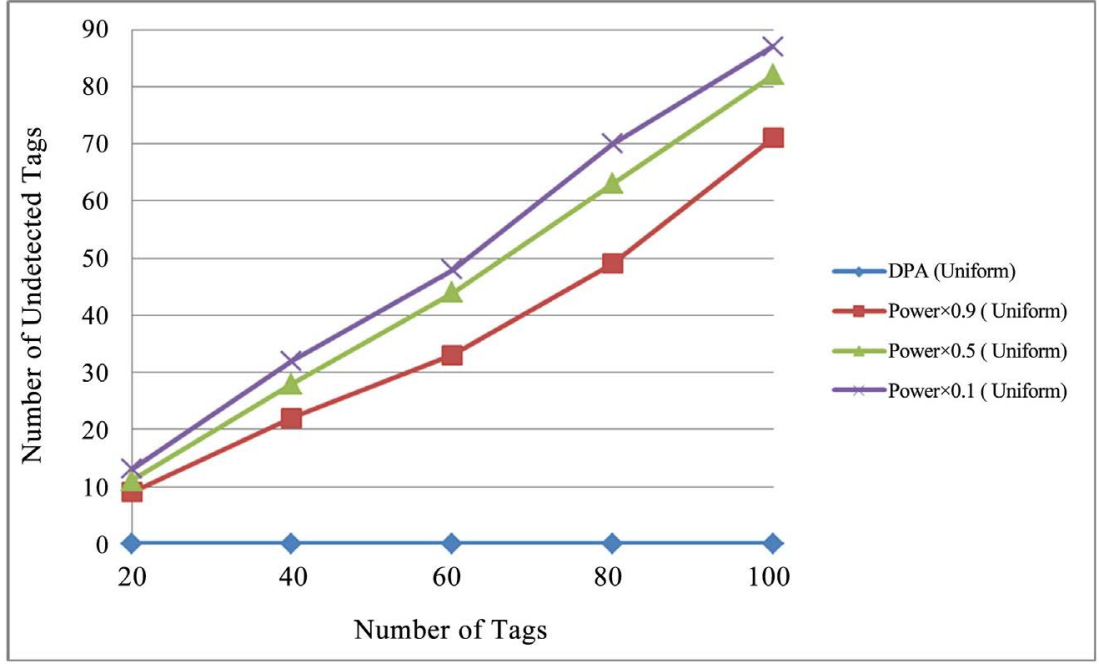

(a)

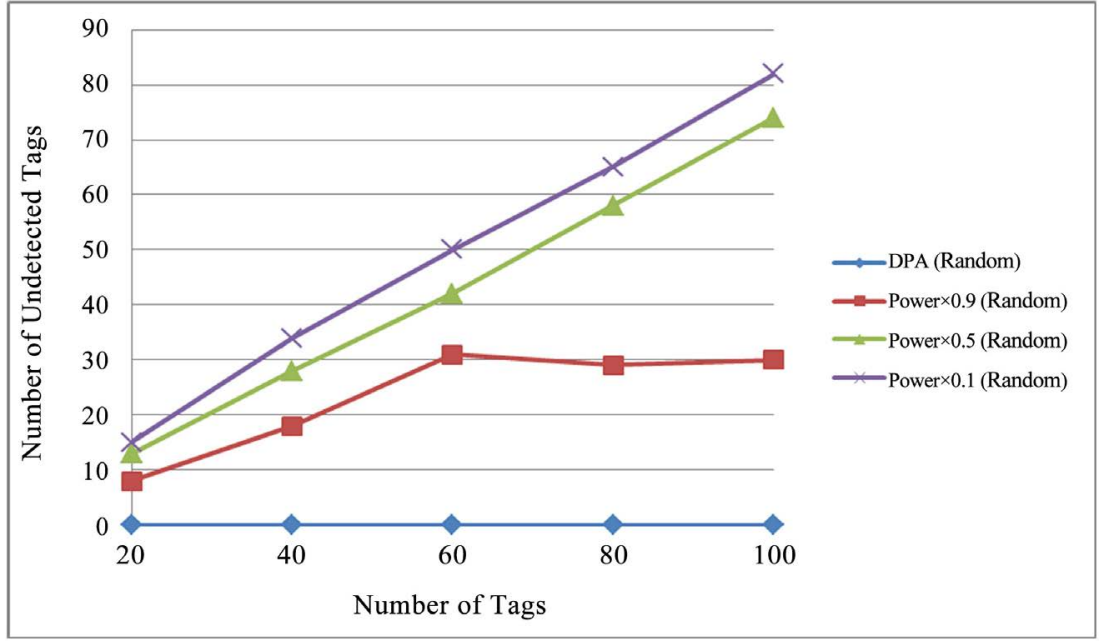

(b)

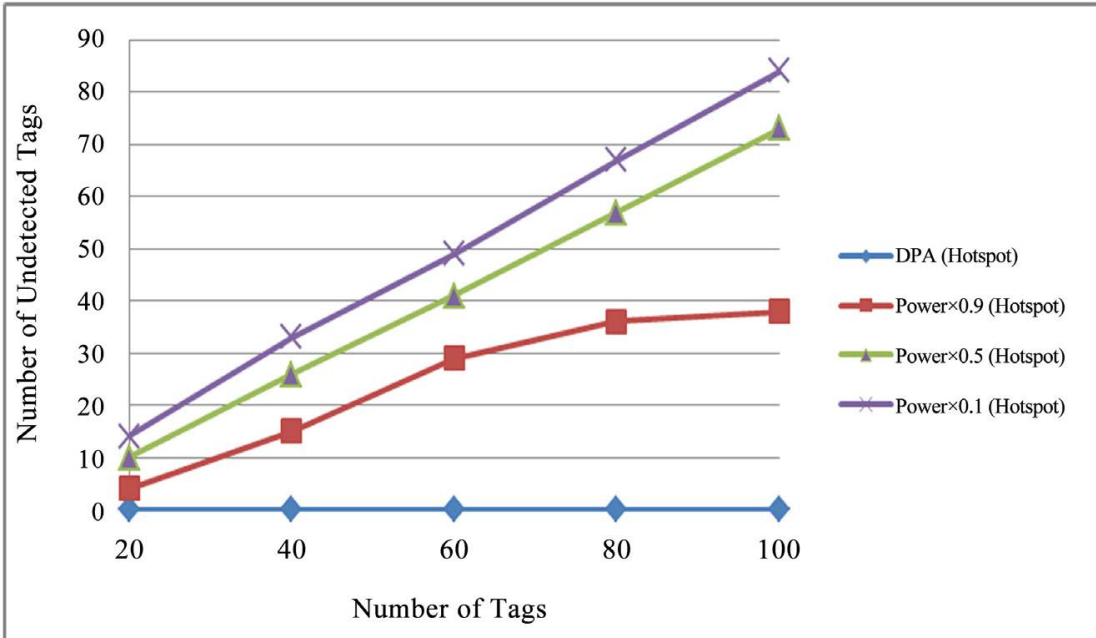

(c)

Figure 10. Comparison of the undetected tags in three distributions. (a) Uniform distribution; (b) Random distribution; (c) Hotspot distribution. 


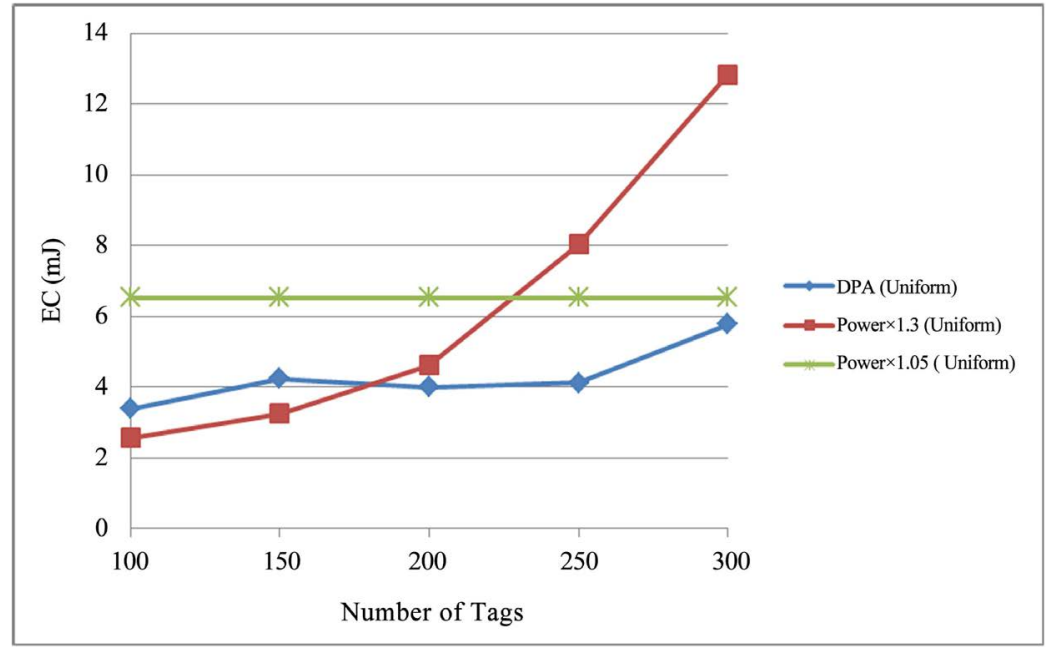

(a)

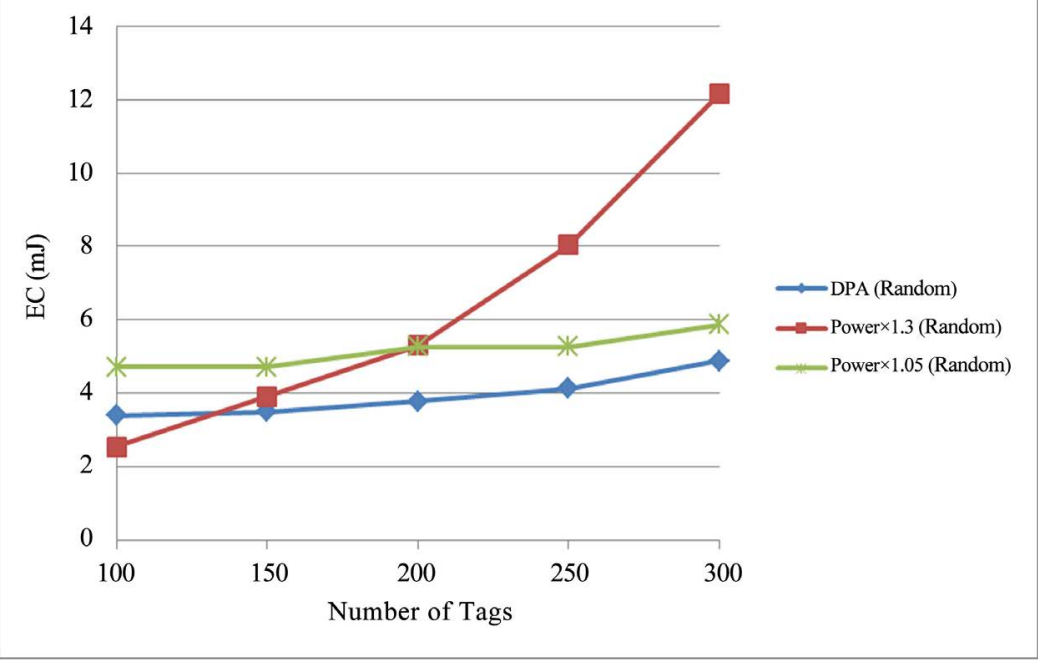

(b)

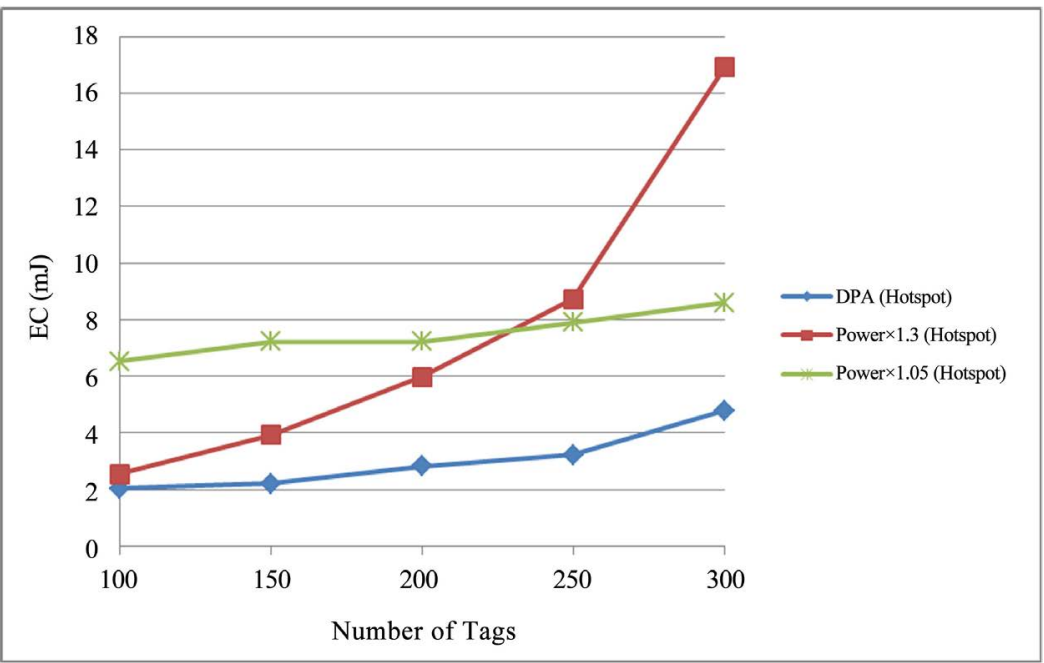

(c)

Figure 11. Comparison in energy consumption for three distributions. (a) Uniform distribution; (b) Random distribution; (c) Hotspot distribution. 
In the future, the proposed DPA can be extended by considering the possible movement of tags. Since a tag may move randomly in every direction, a hop-spot area may move from inner circle to outer circle or vice versa along with the movement of tags. Therefore, how to systematically predict the movement of tags thus dynamically enlarging or shrinking the power coverage would become possible.

\section{Conflicts of Interest}

The authors declare no conflicts of interest regarding the publication of this paper.

\section{References}

[1] Yu, G.J., Lee, W. and Du, D.-Z. (2011) Reducing Reader Collision for Mobile RFID. IEEE Transactions on Consumer Electronics, 57, 574-582. https://doi.org/10.1109/TCE.2011.5955194

[2] Leonardo, D., Sanchez, M., Victor, M. and Ramos, R. (2011) p-Persistent CSMA as a Collision Resolution Protocol for Active RFID Environments. Eighth International Conference on Wireless and Optical Communications Networks (WOCN), Paris, 24-26 May 2011, 1-5.

[3] Kim, S., Kwack, S., Choi, S. and Lee, B.G. (2011) Enhanced Collision Arbitration Protocol Utilizing Multiple Antennas in RFID Systems. Asia-Pacific Conference on Communications (APCC), Sabah, 2-5 October 2011, 925-929. https://doi.org/10.1109/APCC.2011.6152942

[4] Onat, I. and Miri, A. (2011) A Tag Count Estimation Algorithm for Dynamic Framed ALOHA Based RFID MAC Protocols. IEEE International Conference on Communications (ICC), Kyoto, 5-9 June 2011, 1-5. https://doi.org/10.1109/icc.2011.5962792

[5] Luo, Z.Y., Shen, Y.F., Wang, H. and Jiang, J. (2011) An Approach of Adjusting Frame Size in Anti-Collision Algorithm for Active RFID System. International Conference on Communication Technology (ICCT), Jinan, 25-28 September 2011, 328-332.

[6] Zhu, L. and Yum, T.-S.P. (2010) The Optimal Reading Strategy for EPC Gen-2 RFID Anti-Collision Systems. IEEE Transactions on Communications, 58, 2725-2733. https://doi.org/10.1109/TCOMM.2010.080310.090421

[7] Zanella, A. (2012) Estimating Collision Set Size in Framed Slotted Aloha Wireless Networks and RFID Systems. IEEE Communications Letters, 16, 300-303. https://doi.org/10.1109/LCOMM.2012.011312.112067

[8] Xu, Y. and Chen, Y.F. (2015) An Improved Dynamic Framed Slotted ALOHA Anti-collision Algorithm based on Estimation Method for RFID Systems. IEEE International Conference on RFID, San Diego, 15-17 April 2015, 1-8.

[9] Wang, S., Hong, W.J., Yin, L. and Li, S.F. (2012) A Novel Fast Tag Estimate Method for Dynamic Frame Length Aloha Anti-Collision Algorithms in RFID System. IEEE Vehicular Technology Conference (VTC Fall), Quebec, 3-6 September 2012, 1-8.

[10] Qian, C., Liu, Y.H., Ngan, H. and Ni, L.M. (2011) Cardinality Estimation for Large-Scale RFID Systems. IEEE Transactions on Parallel and Distributed Systems, 22, 1441-1454. https://doi.org/10.1109/TPDS.2011.36

[11] La Porta, T.F., Maselli, G. and Petrioli, C. (2011) Anticollision Protocols for Sin- 
gle-Reader RFID Systems: Temporal Analysis and Optimization. IEEE Transactions on Mobile Computing, 10, 267-279. https://doi.org/10.1109/TMC.2010.58

[12] La Porta, T.F., Maselli, G. and Petrioli, C. (2009) Optimal Frame Tuning for Aloha Protocols in RFID Networks. Annual IEEE Communications Society Conference on Sensor, Mesh and Ad Hoc Communications and Networks Workshops, SECON Workshops, Rome, June 2009, 1-3. https://doi.org/10.1109/SAHCNW.2009.5172951

[13] Wu, H.F. and Zeng, Y. (2011) Efficient Framed Slotted Aloha Protocol for RFID Tag Anticollision. IEEE Transactions on Automation Science and Engineering, 8, 581-588. https://doi.org/10.1109/TASE.2010.2101061

[14] Chen, W.-T. (2012) A New RFID Anti-Collision Algorithm for the EPC Global UHF Class-1 Generation-2 Standard. International Conference on Ubiquitous Intelligence and Computing, Fukuoka, 4-7 September 2012.

[15] Chen, W.-T. and Kao, W.-B. (2011) A Novel Q-Algorithm for EPC Global Class-1 Generation-2 Anti-Collision Protocol. World Academy of Science, Engineering and Technology, 78, 801-804.

[16] Chen, W.-T. (2015) Optimal Frame Length Analysis and an Efficient Anti-Collision Algorithm with Early Adjustment of Frame Length for RFID Systems. IEEE Transactions on Vehicular Technology, 1.

[17] Farooq, M.U., Asif, M., Nabi, S.W. and Qureshi, M.A. (2012) Optimal Adjustment Parameters for EPC Global RFID Anti-Collision Q-Algorithm in Different Traffic Scenarios. International Conference on Frontiers of Information Technology, Islamabad, 17-19 December 2012, 302-305. https://doi.org/10.1109/FIT.2012.61

[18] Qiao, J., Wang, W. and Zhang, Y. (2011) Matching Grouping Dynamic Frame Slotted ALOHA for Anti-Collision in RFID Systems. IET International Conference on Communication Technology and Application, Beijing, 14-16 October 2011.

[19] Wang, C.-Y. and Lee, C.-C. (2010) A Grouping-Based Dynamic Framed Slotted ALOHA Anti-Collision Method with Fine Groups in RFID Systems. International Conference on Future Information Technology, Busan, 21-23 May 2010, 1-5. https://doi.org/10.1109/FUTURETECH.2010.5482699

[20] Su, Y.-S. and Tonguz, O.K. (2013) Using the Chinese Remainder Theorem for the Grouping of RFID Tags. IEEE Transactions on Communications, 61, 4741-4753. https://doi.org/10.1109/TCOMM.2013.101113.130216

[21] Tseng, D.F. and Lin, Z.C. (2009) Anti-Collision Algorithm with the Aid of Interference Cancellation and Tag Set Partitioning in Radio-Frequency Identification Systems. IET Communications, 3, 143-150. https://doi.org/10.1049/iet-com:20070524

[22] EM4100 Protocol (2004) EM Microelectronic-Marin SA. 